\title{
SOME KINDS OF SHADOWING PROPERTIES OF NON-AUTONOMOUS PRODUCT SYSTEMS
}

\author{
JINGMIN PI ${ }^{1}$, TIANXIU LU ${ }^{1, *}$, XIAOFANG YANG ${ }^{1,2}$ AND YONGXI JIANG ${ }^{1,2}$
}

\begin{abstract}
This paper proved that the product system $\overline{f_{1, \infty}} \times \overline{g_{1, \infty}}$ has pseudo-orbit shadowing property (resp., average shadowing property, asymptotic average shadowing property, weak asymptotic average shadowing property) if and only if $\overline{1_{1, \infty}}$ and $\overline{g_{1, \infty}}$ have (resp., average shadowing property, asymptotic average shadowing property, weak asymptotic average shadowing property), where $\left(\overline{f_{n}}\right)_{n=1}^{\infty},\left(\overline{g_{n}}\right)_{n=1}^{\infty}$ is the continuous mapping sequences on $\mathcal{K}(X)$ and $\mathcal{K}(Y)$, respectively.
\end{abstract}

\section{INTRODUCTION}

Shadowing property is an important research direction in dynamical systems. In recent years, the research on shadowing in dynamic systems has been carried out continuously. Erivelton ${ }^{[1]}$ reported the existence of multiple pseudo-orbits in the setup of using digital computers to simulate different continuous nonlinear systems. Zhan ${ }^{[2]}$ focused on the existence of true solutions near the numerical approximate solutions of stochastic differential equations that can produce stochastic dynamic systems. Lucas ${ }^{[3]}$ introduced a method for solving nonlinear differential equations. That is to construct a linear differential equation and use the pseudo-orbital solution to approximate the true solution. Kawaguchi ${ }^{[4]}$ gave sufficient conditions that a point can be approximated by an entropy point in terms of the sensitivity and the shadowing property. The chaoticity of the shadowing system, such as ergodicity, topological entropy, sensitivity, have also attracted the attention of many dynamic system researcher ${ }^{[4-12]}$. Inspired by these thinking, we discuss some shadowing properties of the product system $\overline{f_{1, \infty}} \times \overline{g_{1, \infty}}$.

The conclusions involve four kinds of shadowing properties, which are pseudo-orbit shadowing property, average shadowing property, asymptotic average shadowing property, and weak asymptotic average shadowing property. It is proved that $\overline{f_{1, \infty}} \times \overline{g_{1, \infty}}$ has these properties if and only if $\overline{f_{1, \infty}}$ and $\overline{g_{1, \infty}}$ have these properties.

\footnotetext{
${ }^{1}$ College of Mathematics and Statistics, Sichuan University of Science and Engineering, ZigOng, 643000, P.R. ChinA;

${ }^{2}$ Key Laboratory of Higher Education of Sichuan Province for Enterprise InformationalIZATION AND Internet of Things, Zigong, 643000, ChinA

*Corresponding aUthor

E-mail addresses: pi1011225770@126.com, lubeeltx@163.com, yxf_suse@163.com, jyx9708170163.com.

Key words and phrases. pseudo-orbit; product system; shadowing property.

Received 22/09/2021.
} 


\section{Preliminaries}

Let $X=[0,1], \mathbb{N}=\{1,2, \cdots\}$. The metric on $\mathrm{X}$ is denoted by $\rho . f_{n}: X \rightarrow X, n \in \mathbb{N}$ is a mapping sequence, and denoted by $f_{1, \infty}=\left(f_{1}, f_{2}, \cdots\right)=\left(\overline{f_{n}}\right)_{n=1}^{\infty}$. This sequence defines a non-autonomous discrete system $\left(X, f_{1, \infty}\right)$. Under this mapping sequence, the orbit of the point $x \in X$ is $\operatorname{Orb}_{f_{1, \infty}}=\left(f_{1}^{n}(x)\right)(n \in \mathbb{N})$, where $f_{1}^{n}=f_{n} \circ \cdots \circ f_{1}, f_{1}^{0}$ denotes the identity mapping. Similarly, $f_{n}^{k}=f_{n+k-1} \circ \cdots \circ f_{n+1} \circ f_{n}$.

Let $\mathcal{K}(X)$ be the hyperspace on $\mathrm{X}$, that is, the system $\left(X, f_{1, \infty}\right)$ induces a set-valued dynamical system $\left(\mathcal{K}(X), f_{1, \infty}\right)$. Where $\overline{f_{1, \infty}}: \mathcal{K}(X) \longrightarrow \mathcal{K}(X)$ is defined as for any $A \in \mathcal{K}(X)$.

Definition 2.1 Let $\delta>0$ and $\left\{x_{i}\right\}_{i=1}^{+\infty} \subset X$

(1) The sequence $\left\{x_{i}\right\}_{i=1}^{+\infty} \subset X$ is $\delta$ pseudo-orbit of $f_{1, \infty}$, if $d\left(f_{i}\left(x_{i}\right), x_{i+1}\right)<\delta$ for any $i \in \mathbb{N}$.

(2) The sequence $\left\{x_{i}\right\}_{i=1}^{+\infty}$ is $\delta$ average pseudo-orbit of $f_{1, \infty}$, if there exists a $n \in \mathbb{N}$ such that for any $n \geq \mathbb{N}$ and $k \in \mathbb{N}_{0}=\mathbb{N} \cup\{0\}$, one has that

$$
\frac{1}{n} \sum_{i=1}^{n} d\left(f_{i}\left(x_{i+k}\right), x_{i+k+1}\right)<\delta .
$$

(3) The sequence $\left\{x_{i}\right\}_{i=1}^{+\infty}$ is the asymptotic average pseudo-orbits of $f_{1, \infty}$, if it satisfying

$$
\lim _{n \rightarrow+\infty} \frac{1}{n} \sum_{i=1}^{n} d\left(f_{i}\left(x_{i}\right), x_{i+1}\right) .
$$

Definition 2.2 (1) A orbit of $f_{1, \infty}$ can be $\varepsilon-$ shadowed by $f$, if for any $x \in X$ and any $i \in \mathbb{N}$,

$$
d\left(f_{i}(x), f_{1}^{i}(x)\right)<\varepsilon .
$$

(2) The system $\left(X, f_{1, \infty}\right)$ has pseudo-orbit shadowing property if for any $\varepsilon>0$ there exists a $\delta>0$ such that for any $\delta$ pseudo-orbit $\left\{x_{i}\right\}_{i=1}^{+\infty}$ of $\left(X, f_{1, \infty}\right)$, there exists a $z \in X$ satisfying $d\left(f_{1}^{i}(z), x_{i}\right)<\varepsilon$ for any $i \in \mathbb{N}$.

(3) The system $\left(X, f_{1, \infty}\right)$ has average shadowing property if for any $\varepsilon>0$ there exists a $\delta>0$ such that for any $\delta$ average pseudo-orbits $\left\{x_{i}\right\}_{i=1}^{+\infty}$ of $f_{1, \infty}$, there exists a $z \in X$ such that

$$
\lim _{n \rightarrow \infty} \sup \frac{1}{n} \sum_{i=1}^{n} d\left(f_{1}^{i}(z), x_{i}\right)<\varepsilon .
$$

(4) The system $\left(X, f_{1, \infty}\right)$ has asymptotic average shadowing property if each asymptotic average pseudo-orbits $\left\{x_{i}\right\}_{i=1}^{+\infty}$ can be tracked progressively by a certain point $z \in X$, that is

$$
\lim _{n \rightarrow \infty} \frac{1}{n} \sum_{i=1}^{n} d\left(f_{1}^{i}(z), x_{i}\right)=0 .
$$

(5) The system $\left(X, f_{1, \infty}\right)$ has weak asymptotic average shadowing property if for any $\varepsilon>0$ and any asymptotic average pseudo-orbits $\left\{x_{i}\right\}_{i=1}^{+\infty}$, there exists a $z \in X$ can $\varepsilon$ - average shadowing $\left\{x_{i}\right\}_{i=1}^{+\infty}$, that is

$$
\lim _{n \rightarrow \infty} \sup \frac{1}{n} \sum_{i=1}^{n} d\left(f_{1}^{i}(z), x_{i}\right)<\varepsilon .
$$


3. Shadowing Property of the Mapping Sequences and the Product Operation

Let $\left(\overline{f_{n}}\right)_{n=1}^{\infty},\left(\overline{g_{n}}\right)_{n=1}^{\infty}$ is the continuous mapping sequences on $\mathcal{K}(X)$ and $\mathcal{K}(Y)$, for any $\left\{A_{i}\right\}_{i \in \mathbb{N}} \subset \mathcal{K}(X),\left\{B_{i}\right\}_{i \in \mathbb{N}} \subset \mathcal{K}(Y)$ and $i, K \in \mathbb{N}$, let

$$
D\left(\overline{f_{i}} \times \overline{g_{i}}\left(A_{i+k}, B_{i+k}\right),\left(A_{i+k+1}, B_{i+k+1}\right)\right)=\max \left\{H_{1}\left(\overline{f_{i}}\left(A_{i+k}\right), A_{i+k+1}\right), H_{2}\left(\overline{g_{i}}\left(B_{i+k}\right), B_{i+k+1}\right)\right\}
$$

is the metric on $\mathcal{K}(X) \times \mathcal{K}(Y)$.

Theorem 3.1 Let $\left(\overline{f_{n}}\right)_{n=1}^{\infty},\left(\overline{g_{n}}\right)_{n=1}^{\infty}$ is the continuous mapping sequences on $\mathcal{K}(X)$ and $\mathcal{K}(Y)$, respectively. Then $\overline{f_{1, \infty}} \times \overline{g_{1, \infty}}$ has pseudo-orbit shadowing property if and only if $\overline{f_{1, \infty}}$ and $\overline{g_{1, \infty}}$ have pseudo-orbit shadowing property.

Proof.(Necessity) Suppose that $\overline{f_{1, \infty}} \times \overline{g_{1, \infty}}$ has pseudo-orbit shadowing property. Let $\left\{A_{i}\right\}_{i \in \mathbb{N}} \subset \mathcal{K}(X),\left\{B_{i}\right\}_{i \in \mathbb{N}} \subset \mathcal{K}(Y)$ is the $\delta$ pseudo-orbit of $\overline{f_{1, \infty}}$ and $\overline{g_{1, \infty}}$, respectively. For any $i \in \mathbb{N}$, one has that

$$
H_{1}\left(\overline{f_{i}}\left(A_{i}\right), A_{i+1}\right)<\delta \quad \text { and } \quad H_{2}\left(\overline{g_{i}}\left(B_{i}\right), B_{i+1}\right)<\delta .
$$

Then, for any $i \in \mathbb{N}$, one has that

$$
\begin{aligned}
& D\left(\overline{f_{i}} \times \overline{g_{i}}\left(A_{i+k}, B_{i+k}\right),\left(A_{i+k+1}, B_{i+k+1}\right)\right) \\
& =\max \left\{H_{1}\left(\overline{f_{i}}\left(A_{i+k}\right), A_{i+k+1}\right), H_{2}\left(\overline{g_{i}}\left(B_{i+k}\right), B_{i+k+1}\right)\right\} \\
& <\delta .
\end{aligned}
$$

So, $\left\{\left(A_{i}, B_{i}\right)\right\}_{i \in \mathbb{N}}$ is the $\delta$ pseudo-orbit of $\overline{f_{1, \infty}} \times \overline{g_{1, \infty}}$. By the pseudo-orbit shadowing property of $\overline{f_{1, \infty}} \times \overline{g_{1, \infty}}$, for any $\varepsilon>0, i \in \mathbb{N}$ there exists $(A, B) \in \mathcal{K}(X) \times \mathcal{K}(Y)$, such that

$$
\begin{aligned}
& D\left(\overline{f_{1, \infty}} \times \overline{g_{1, \infty}}(A, B),\left(A_{i}, B_{i}\right)\right) \\
& =\max \left\{H_{1}\left(\overline{f_{1}^{i}}(A), A_{i}\right), H_{2}\left(\overline{g_{1}^{i}}(B), B_{i}\right)\right\} \\
& <\varepsilon .
\end{aligned}
$$

This implies that

$$
H_{1}\left(\overline{f_{1}^{i}}(A), A_{i}\right)<\varepsilon \quad \text { and } \quad H_{2}\left(\overline{g_{1}^{i}}(B), B_{i}\right) .
$$

Thus, $\overline{f_{1, \infty}}$ and $\overline{g_{1, \infty}}$ have pseudo-orbit shadowing property.

(Sufficiency) Assume that $\overline{f_{1, \infty}}$ and $\overline{g_{1, \infty}}$ have pseudo-orbit shadowing property and let $\left\{\left(A_{i}, B_{i}\right)\right\}_{i \in \mathbb{N}} \subset \mathcal{K}(X) \times \mathcal{K}(Y)$ is the $\delta$ pseudo-orbit of $\overline{f_{1, \infty}} \times \overline{g_{1, \infty}}$. Then for any $i \in \mathbb{N}$, one has that

$$
\begin{aligned}
& D\left(\overline{f_{i}} \times \overline{g_{i}}\left(A_{i}, B_{i}\right),\left(A_{i+1}, B_{i+1}\right)\right) \\
& =\max \left\{H_{1}\left(\overline{f_{i}}\left(A_{i}\right), A_{i+1}\right), H_{2}\left(\overline{g_{i}}\left(B_{i}\right), B_{i+1}\right)\right\} \\
& <\delta .
\end{aligned}
$$

Then

$$
H_{1}\left(\overline{f_{i}}\left(A_{i}\right), A_{i+1}<\delta \quad \text { and } \quad H_{2}\left(\overline{g_{i}}\left(B_{i}\right), B_{i+1}\right) .\right.
$$

So, $\left\{A_{i}\right\}_{i \in \mathbb{N}},\left\{B_{i}\right\}_{i \in \mathbb{N}}$ is the $\delta$ pseudo-orbit of $\overline{f_{1, \infty}}$ and $\overline{g_{1, \infty}}$, respectively. According to the pseudo-orbit shadowing propertivity of $\overline{f_{1, \infty}}$ and $\overline{g_{1, \infty}}$, it follows that for any $\varepsilon>0, i \in \mathbb{N}$ there exists $A \in \mathcal{K}(X), B \in \mathcal{K}(Y)$, such that

$$
H_{1}\left(\overline{f_{1}^{i}}(A), A_{i}\right)<\varepsilon \quad \text { and } \quad H_{2}\left(\overline{g_{1}^{i}}(B), B_{i}\right) .
$$


Thus

$$
\begin{aligned}
& D\left(\overline{f_{1, \infty}} \times \overline{g_{1, \infty}}(A, B),\left(A_{i}, B_{i}\right)\right) \\
& =\max \left\{H_{1}\left(\overline{f_{1}^{i}}(A), A_{i}\right), H_{2}\left(\overline{g_{1}^{i}}(B), B_{i}\right)\right\} \\
& <\varepsilon .
\end{aligned}
$$

So, $\overline{f_{1, \infty}} \times \overline{g_{1, \infty}}$ has pseudo-orbit shadowing property.

Theorem 3.2 Let $\left(\overline{f_{n}}\right)_{n=1}^{\infty},\left(\overline{g_{n}}\right)_{n=1}^{\infty}$ is the continuous mapping sequences on $\mathcal{K}(X)$ and $\mathcal{K}(Y)$, respectively. Then $\overline{f_{1, \infty}} \times \overline{g_{1, \infty}}$ has average shadowing property if and only if $\overline{f_{1, \infty}}$ and $\overline{g_{1, \infty}}$ have average shadowing property.

Proof.(Necessity) Suppose that $\overline{f_{1, \infty}} \times \overline{g_{1, \infty}}$ has average shadowing property. Let $\left\{A_{i}\right\}_{i \in \mathbb{N}} \subset$ $\mathcal{K}(X),\left\{B_{i}\right\}_{i \in \mathbb{N}} \subset \mathcal{K}(Y)$ is the $\delta$ pseudo-orbit of $\overline{f_{1, \infty}}$ and $\overline{g_{1, \infty}}$, respectively. Then there exist $N_{1}, N_{2}>0$ such that for any $n_{1}>N_{1}, n_{2}>N_{2}$, and any $k \in \mathbb{N}$, one has that

$$
\frac{1}{n_{1}} \sum_{i=1}^{n_{1}} H_{1}\left(\overline{f_{i}}\left(A_{i+k}\right), A_{i+k+1}\right)<\delta \quad \text { and } \quad \frac{1}{n_{2}} \sum_{i=1}^{n_{2}} H_{2}\left(\overline{g_{i}}\left(B_{i+k}\right), B_{i+k+1}\right)<\delta .
$$

Take $N=\max \left\{N_{1}, N_{2}\right\}$, for any $n>N$ and above $k \in \mathbb{N}_{0}$, one has that

$$
\begin{aligned}
& \frac{1}{n} \sum_{i=1}^{n} D\left(\overline{f_{i}} \times \overline{g_{i}}\left(A_{i+k}, B_{i+k}\right),\left(A_{i+k+1}, B_{i+k+1}\right)\right) \\
& =\frac{1}{n} \sum_{i=1}^{n} \max \left\{H_{1}\left(\overline{f_{i}}\left(A_{i+k}\right), A_{i+k+1}\right), H_{2}\left(\overline{g_{i}}\left(B_{i+k}\right), B_{i+k+1}\right)\right\} \\
& =\max \left\{\frac{1}{n} \sum_{i=1}^{n} H_{1}\left(\overline{f_{i}}\left(A_{i+k}\right), A_{i+k+1}\right), \frac{1}{n} \sum_{i=1}^{n} H_{2}\left(\overline{g_{i}}\left(B_{i+k}\right), B_{i+k+1}\right)\right\} \\
& <\delta .
\end{aligned}
$$

So, $\left\{\left(A_{i}, B_{i}\right)\right\}_{i \in \mathbb{N}}$ is the $\delta$ average pseudo-orbit of $\overline{f_{1, \infty}} \times \overline{g_{1, \infty}}$. By the average shadowing property of $\overline{f_{1, \infty}} \times \overline{g_{1, \infty}}$, for any $\varepsilon>0$, there exists $(A, B) \in \mathcal{K}(X) \times \mathcal{K}(Y)$, such that

$$
\begin{aligned}
& \lim _{n \rightarrow \infty} \sup \frac{1}{n} \sum_{i=1}^{n} D\left(\overline{f_{1, \infty}} \times \overline{g_{1, \infty}}(A, B),\left(A_{i}, B_{i}\right)\right) \\
& =\lim _{n \rightarrow \infty} \sup \frac{1}{n} \sum_{i=1}^{n} \max \left\{H_{1}\left(\overline{f_{1}^{i}}(A), A_{i}\right), H_{2}\left(\overline{g_{1}^{i}}(B), B_{i}\right)\right\} \\
& <\varepsilon .
\end{aligned}
$$

This implies that

$$
\lim _{n \rightarrow \infty} \sup \frac{1}{n} \sum_{i=1}^{n} H_{1}\left(\overline{f_{1}^{i}}(A), A_{i}\right)<\varepsilon \quad \text { and } \quad \lim _{n \rightarrow \infty} \sup \frac{1}{n} \sum_{i=1}^{n} H_{2}\left(\overline{g_{1}^{i}}(B), B_{i}\right)<\varepsilon .
$$

Thus, $\overline{f_{1, \infty}}$ and $\overline{g_{1, \infty}}$ have average shadowing property. 
(Sufficiency) Assume that $\overline{f_{1, \infty}}$ and $\overline{g_{1, \infty}}$ have average shadowing property and let $\left\{\left(A_{i}, B_{i}\right)\right\}_{i \in \mathbb{N}} \subset \mathcal{K}(X) \times \mathcal{K}(Y)$ is the $\delta$ average pseudo-orbit of $\overline{f_{1, \infty}} \times \overline{g_{1, \infty}}$. Then there exists a $N>0$, for any $n \geq \mathbb{N}$ and $k \in \mathbb{N}$, one has that

$$
\begin{aligned}
& \frac{1}{n} \sum_{i=1}^{n} D\left(\overline{f_{i}} \times \overline{g_{i}}\left(A_{i+k}, B_{i+k}\right),\left(A_{i+k+1}, B_{i+k+1}\right)\right) \\
& =\frac{1}{n} \sum_{i=1}^{n} \max \left\{H_{1}\left(\overline{f_{i}}\left(A_{i+k}\right), A_{i+k+1}\right), H_{2}\left(\overline{g_{i}}\left(B_{i+k}\right), B_{i+k+1}\right)\right\} \\
& <\delta
\end{aligned}
$$

Then

$$
\frac{1}{n} \sum_{i=1}^{n} H_{1}\left(\overline{f_{i}}\left(A_{i+k}\right), A_{i+k+1}\right)<\delta \quad \text { and } \quad \frac{1}{n} \sum_{i=1}^{n} H_{2}\left(\overline{g_{i}}\left(B_{i+k}\right), B_{i+k+1}\right)<\delta
$$

So, $\left\{A_{i}\right\}_{i \in \mathbb{N}},\left\{B_{i}\right\}_{i \in \mathbb{N}}$ is the $\delta$ average pseudo-orbit of $\overline{f_{1, \infty}}$ and $\overline{g_{1, \infty}}$, respectively. According to the average shadowing propertivity of $\overline{f_{1, \infty}}$ and $\overline{g_{1, \infty}}$, it follows that for any $\varepsilon>0$, there exists $A \in \mathcal{K}(X), B \in \mathcal{K}(Y)$, such that

$$
\lim _{n \rightarrow \infty} \sup \frac{1}{n} \sum_{i=1}^{n} H_{1}\left(\overline{f_{i}}\left(A_{i}\right), A_{i+1}\right)<\delta \quad \text { and } \quad \lim _{n \rightarrow \infty} \sup \frac{1}{n} \sum_{i=1}^{n} H_{2}\left(\overline{g_{i}}\left(B_{i}\right), B_{i+1}\right)<\delta
$$

Thus one has that

$$
\begin{aligned}
& \lim _{n \rightarrow \infty} \sup \frac{1}{n} \sum_{i=1}^{n} D\left(\overline{f_{1, \infty}} \times \overline{g_{1, \infty}}(A, B),\left(A_{i}, B_{i}\right)\right) \\
& =\lim _{n \rightarrow \infty} \sup \frac{1}{n} \sum_{i=1}^{n} \max \left\{H_{1}\left(\overline{f_{1}^{i}}(A), A_{i}\right), H_{2}\left(\overline{g_{1}^{i}}(B), B_{i}\right)\right\} \\
& <\varepsilon .
\end{aligned}
$$

So, $\overline{f_{1, \infty}} \times \overline{g_{1, \infty}}$ has average shadowing property.

Theorem 3.3 Let $\left(\overline{f_{n}}\right)_{n=1}^{\infty},\left(\overline{g_{n}}\right)_{n=1}^{\infty}$ is the continuous mapping sequences on $\mathcal{K}(X)$ and $\mathcal{K}(Y)$, respectively. Then $\overline{f_{1, \infty}} \times \overline{g_{1, \infty}}$ has asymptotic average shadowing property if and only if $\overline{f_{1, \infty}}$ and $\overline{g_{1, \infty}}$ have asymptotic average shadowing property.

Proof.(Necessity) Suppose that $\overline{f_{1, \infty}} \times \overline{g_{1, \infty}}$ has asymptotic average shadowing property. Let $\left\{A_{i}\right\}_{i \in \mathbb{N}} \subset \mathcal{K}(X),\left\{B_{i}\right\}_{i \in \mathbb{N}} \subset \mathcal{K}(Y)$ is the asymptotic pseudo-orbit of $\overline{f_{1, \infty}}$ and $\overline{g_{1, \infty}}$, respectively. Then

$$
\lim _{n \rightarrow \infty} \frac{1}{n} \sum_{i=1}^{n} H_{1}\left(\overline{f_{i}}\left(A_{i}\right), A_{i+1}\right)<\delta \quad \text { and } \quad \lim _{n \rightarrow \infty} \frac{1}{n} \sum_{i=1}^{n} H_{2}\left(\overline{g_{i}}\left(B_{i}\right), B_{i+1}\right)<\delta
$$

For any $\varepsilon>0$, there exist $N_{1}, N_{2}>0$, such that

$$
\frac{1}{n_{1}} \sum_{i=1}^{n_{1}} H_{1}\left(\overline{f_{i}}\left(A_{i}\right), A_{i+1}\right)<\varepsilon \quad \text { and } \quad \frac{1}{n_{2}} \sum_{i=1}^{n_{2}} H_{2}\left(\overline{g_{i}}\left(B_{i}\right), B_{i+1}\right)<\varepsilon
$$

for any $n_{1}>N_{1}, n_{2}>N_{2}$.

Put $N=\max \left\{N_{1}, N_{2}\right\}$, for any $n>N$, one has that

$$
\max \left\{\frac{1}{n} \sum_{i=1}^{n} H_{1}\left(\overline{f_{i}}\left(A_{i}\right), A_{i+1}\right), \frac{1}{n} \sum_{i=1}^{n} H_{2}\left(\bar{g}_{i}\left(B_{i}\right), B_{i+1}\right)\right\}<\varepsilon
$$


This implies that

$$
\begin{aligned}
& \lim _{n \rightarrow \infty} \frac{1}{n} \sum_{i=1}^{n} D\left(\overline{f_{i}} \times \overline{g_{i}}\left(A_{i}, B_{i}\right),\left(A_{i+1}, B_{i+1}\right)\right) \\
& =\lim _{n \rightarrow \infty} \max \left\{\frac{1}{n} \sum_{i=1}^{n} H_{1}\left(\overline{f_{i}}\left(A_{i}\right), A_{i+1}\right), \frac{1}{n} \sum_{i=1}^{n} H_{2}\left(\overline{g_{i}}\left(B_{i}\right), B_{i+1}\right)\right\} \\
& =0 .
\end{aligned}
$$

Hence, $\left\{\left(A_{i}, B_{i}\right)\right\}_{i \in \mathbb{N}}$ is the asymptotic average pseudo-orbit of $\overline{f_{1, \infty}} \times \overline{g_{1, \infty}}$. According to the asymptotic average shadowing property of $\overline{f_{1, \infty}} \times \overline{g_{1, \infty}}$, there exists $(A, B) \in \mathcal{K}(X) \times \mathcal{K}(Y)$, such that

$$
\begin{aligned}
& \lim _{n \rightarrow \infty} \frac{1}{n} \sum_{i=1}^{n} D\left(\overline{f_{1, \infty}} \times \overline{g_{1, \infty}}(A, B),\left(A_{i}, B_{i}\right)\right) \\
& =\lim _{n \rightarrow \infty} \frac{1}{n} \sum_{i=1}^{n} \max \left\{H_{1}\left(\overline{f_{1}^{i}}(A), A_{i}\right), H_{2}\left(\overline{g_{1}^{i}}(B), B_{i}\right)\right\} \\
& =0 .
\end{aligned}
$$

Combined with $\frac{1}{n} \sum_{i=1}^{n} H_{1}\left(\overline{f_{1}^{i}}(A), A_{i}\right) \geq 0 \quad$ and $\quad \frac{1}{n} \sum_{i=1}^{n} H_{2}\left(\overline{g_{1}^{i}}(B), B_{i}\right) \geq 0$. for any $\varepsilon>0$, there exists $N>0$ such that

$$
\frac{1}{n} \sum_{i=1}^{n} H_{1}\left(\overline{f_{1}^{i}}(A), A_{i}\right)<\varepsilon \quad \text { and } \quad \frac{1}{n} \sum_{i=1}^{n} H_{2}\left(\overline{g_{1}^{i}}(B), B_{i}\right)<\varepsilon
$$

for any $n>N$.

That is,

$$
\lim _{n \rightarrow \infty} \frac{1}{n} \sum_{i=1}^{n} H_{1}\left(\overline{f_{1}^{i}}(A), A_{i}\right)=0 \quad \text { and } \quad \lim _{n \rightarrow \infty} \frac{1}{n} \sum_{i=1}^{n} H_{2}\left(\overline{g_{1}^{i}}(B), B_{i}\right)=0
$$

So, $\overline{f_{1, \infty}}$ and $\overline{g_{1, \infty}}$ have asymptotic average shadowing property.

(Sufficiency) Assume that $\overline{f_{1, \infty}}$ and $\overline{g_{1, \infty}}$ have asymptotic average shadowing property. let $\left\{\left(A_{i}, B_{i}\right)\right\}_{i \in \mathbb{N}}$ is the asymptotic average pseudo-orbit of $\overline{f_{1, \infty}} \times \overline{g_{1, \infty}}$. Then

$$
\begin{aligned}
& \lim _{n \rightarrow \infty} \frac{1}{n} \sum_{i=1}^{n} D\left(\overline{f_{i}} \times \overline{g_{i}}\left(A_{i}, B_{i}\right),\left(A_{i+1}, B_{i+1}\right)\right) \\
& \left.=\lim _{n \rightarrow \infty} \frac{1}{n} \sum_{i=1}^{n} \max \left\{H_{1}\left(\overline{f_{i}}\left(A_{i}\right), A_{i+1}\right), H_{2}\left(\overline{g_{i}}\left(B_{i}\right), B_{i+1}\right)\right\}\right\} \\
& =0
\end{aligned}
$$

So,

$$
\lim _{n \rightarrow \infty} \frac{1}{n} \sum_{i=1}^{n} H_{1}\left(\overline{f_{i}}\left(A_{i}\right), A_{i+1}\right)=0 \quad \text { and } \quad \lim _{n \rightarrow \infty} \frac{1}{n} \sum_{i=1}^{n} H_{2}\left(\overline{g_{i}}\left(B_{i}\right), B_{i+1}\right)=0
$$

It follows that $\left\{A_{i}\right\}_{i \in \mathbb{N}},\left\{B_{i}\right\}_{i \in \mathbb{N}}$ is the asymptotic average pseudo-orbit of $\overline{f_{1, \infty}}$ and $\overline{g_{1, \infty}}$, respectively. there exists $A \in \mathcal{K}(X), B \in \mathcal{K}(Y)$, such that

$$
\lim _{n \rightarrow \infty} \frac{1}{n} \sum_{i=1}^{n} H_{1}\left(\overline{f_{1}^{i}}(A), A_{i}\right)=0 \quad \text { and } \quad \lim _{n \rightarrow \infty} \frac{1}{n} \sum_{i=1}^{n} H_{2}\left(\overline{g_{1}^{i}}(B), B_{i}\right)=0
$$


And since

$$
\begin{aligned}
& \lim _{n \rightarrow \infty} \frac{1}{n} \sum_{i=1}^{n} D\left(\overline{f_{1, \infty}} \times \overline{g_{1, \infty}}(A, B),\left(A_{i}, B_{i}\right)\right) \\
& =\lim _{n \rightarrow \infty} \frac{1}{n} \sum_{i=1}^{n} \max \left\{H_{1}\left(\overline{f_{1}^{i}}(A), A_{i}\right), H_{2}\left(\overline{g_{1}^{i}}(B), B_{i}\right)\right\} \\
& =0 .
\end{aligned}
$$

Thus, $\overline{f_{1, \infty}} \times \overline{g_{1, \infty}}$ has asymptotic average shadowing property.

Theorem 3.4 Let $\left(\overline{f_{n}}\right)_{n=1}^{\infty},\left(\overline{g_{n}}\right)_{n=1}^{\infty}$ is the continuous mapping sequences on $\mathcal{K}(X)$ and $\mathcal{K}(Y)$, respectively. Then $\overline{f_{1, \infty}} \times \overline{g_{1, \infty}}$ has weak asymptotic average shadowing property if and only if $\overline{f_{1, \infty}}$ and $\overline{g_{1, \infty}}$ have weak asymptotic average shadowing property.

Proof.(Necessity) Suppose that $\overline{f_{1, \infty}} \times \overline{g_{1, \infty}}$ has weak asymptotic average shadowing property. Let $\left\{A_{i}\right\}_{i \in \mathbb{N}} \subset \mathcal{K}(X),\left\{B_{i}\right\}_{i \in \mathbb{N}} \subset \mathcal{K}(Y)$ is the asymptotic pseudo-orbit of $\overline{f_{1, \infty}}$ and $\overline{g_{1, \infty}}$, respectively. According to the proof of Theorem 3.3, the orbit $\left\{\left(A_{i}, B_{i}\right)\right\}_{i \in \mathbb{N}}$ is the asymptotic average pseudo-orbit of $\overline{f_{1, \infty}} \times \overline{g_{1, \infty}}$. By the weak asymptotic average shadowing property of $\overline{f_{1, \infty}} \times \overline{g_{1, \infty}}$, there exists $(A, B) \in \mathcal{K}(X) \times \mathcal{K}(Y)$, such that

$$
\begin{aligned}
& \lim _{n \rightarrow \infty} \sup \frac{1}{n} \sum_{i=1}^{n} D\left(\overline{f_{1, \infty}} \times \overline{g_{1, \infty}}(A, B),\left(A_{i}, B_{i}\right)\right) \\
& =\lim _{n \rightarrow \infty} \sup \frac{1}{n} \sum_{i=1}^{n} \max \left\{H_{1}\left(\overline{f_{1}^{i}}(A), A_{i}\right), H_{2}\left(\overline{g_{1}^{i}}(B), B_{i}\right)\right\} \\
& <\varepsilon .
\end{aligned}
$$

It is easy to know that

$$
\lim _{n \rightarrow \infty} \sup \frac{1}{n} \sum_{i=1}^{n} H_{1}\left(\overline{f_{1}^{i}}(A), A_{i}\right)<\varepsilon \quad \text { and } \quad \lim _{n \rightarrow \infty} \sup \frac{1}{n} \sum_{i=1}^{n} H_{2}\left(\overline{g_{1}^{i}}(B), B_{i}\right)<\varepsilon
$$

So, $\overline{f_{1, \infty}}$ and $\overline{g_{1, \infty}}$ have weak asymptotic average shadowing property.

(Sufficiency) Assume that $\overline{f_{1, \infty}}$ and $\overline{g_{1, \infty}}$ have weak asymptotic average shadowing property and let $\left\{\left(A_{i}, B_{i}\right)\right\}_{i \in \mathbb{N}}$ is the asymptotic average pseudo-orbit of $\overline{f_{1, \infty}} \times \overline{g_{1, \infty}}$. Then according to the proof of Theorem 3.3, it follows that $\left\{A_{i}\right\}_{i \in \mathbb{N}},\left\{B_{i}\right\}_{i \in \mathbb{N}}$ is the asymptotic average pseudoorbit of $\overline{f_{1, \infty}}$ and $\overline{g_{1, \infty}}$, respectively. According to the weak asymptotic average shadowing property of $\overline{f_{1, \infty}}$ and $\overline{g_{1, \infty}}$, there exists $A \in \mathcal{K}(X), B \in \mathcal{K}(Y)$, such that

$$
\lim _{n \rightarrow \infty} \sup \frac{1}{n} \sum_{i=1}^{n} H_{1}\left(\overline{f_{1}^{i}}(A), A_{i}\right)<\varepsilon \quad \text { and } \quad \lim _{n \rightarrow \infty} \sup \frac{1}{n} \sum_{i=1}^{n} H_{2}\left(\overline{g_{1}^{i}}(B), B_{i}\right)<\varepsilon
$$

And since

$$
\begin{aligned}
& \lim _{n \rightarrow \infty} \sup \frac{1}{n} \sum_{i=1}^{n} D\left(\overline{f_{1, \infty}} \times \overline{g_{1, \infty}}(A, B),\left(A_{i}, B_{i}\right)\right) \\
& =\lim _{n \rightarrow \infty} \sup \frac{1}{n} \sum_{i=1}^{n} \max \left\{H_{1}\left(\overline{f_{1}^{i}}(A), A_{i}\right), H_{2}\left(\overline{g_{1}^{i}}(B), B_{i}\right)\right\} \\
& <\varepsilon .
\end{aligned}
$$


Thus, $\overline{f_{1, \infty}} \times \overline{g_{1, \infty}}$ has weak asymptotic average shadowing property.

\section{Acknowledgement}

This work was funded by the Opening Project of Key Laboratory of Higher Education of Sichuan Province for Enterprise Informationalization and Internet of Things (No. 2020WZJ01), the Scientific Research Project of Sichuan University of Science and Engineering (No. 2020RC24), and the Graduate student Innovation Fund (Nos. y2021100, cx2020188).

\section{REFERENCES}

[1] E. G. Nepomuceno, E. Mendes, On the analysis of pseudo-orbits of continuous chaotic nonlinear systems simulated using discretization schemes in a digital computer, Chaos Soliton. Fract. (95) 2017 21-32. https: //doi.org/10.1016/j.chaos.2016.12.002.

[2] Q. Y. Zhan, Shadowing orbits of a class of random differential equations, Appl. Numer. Math. 13 (2019) 206-214. https://doi.org/10.1016/j.apnum.2018.10.010.

[3] L. Backes, D. Dragievi, A general approach to nonautonomous shadowing for nonlinear dynamics, B. Sci. Math. 170 (2021) 102996. https://doi.org/10.1016/j.bulsci.2021.102996.

[4] N. Kawaguchi, Entropy points of continuous maps with the sensitivity and the shadowing property, Topol. Appl. 210 (2016) 8-15. https://doi.org/10.1016/j.topol.2016.07.004.

[5] P. Koscielniak, M. Mazur, Chaos and the shadowing property, Topol. Appl. 154 (2007) 2553-2557. https: //doi.org/10.1016/j.topol.2006.06.010.

[6] Y. X. Niu, The average-shadowing property and strong ergodicity, J. Math. Anal. Appl. 376 (2011) 528-534. https://doi.org/10.1016/j.jmaa.2010.11.024.

[7] M. Lee, The ergodic shadowing property from the robust and generic view point, Adv. Differ. Equ. 2014 (2014) 170. https://doi.org/10.1186/1687-1847-2014-170.

[8] S. Y. Pilyugin, B. S. Tikhomirov, Vector fields with the oriented shadowing property, J. Differ. Equ. 248 (2010) 1345-1375. https://doi.org/10.1016/j.jde.2009.09.024.

[9] A. Artigue, B. Carvalho, W. Cordeiro, J. Vieitez, Beyond topological hyperbolicity: The L-shadowing property, J. Differ. Equ. 268 (2020) 3057-3080. https://doi.org/10.1016/j.jde.2019.09.052.

[10] J. J. Park, Y. Zhang, Average shadowing properties on compact metric spaces, J. Korean Math. Soc. 21 (2006) 355-361. https://doi.org/10.4134/CKMS . 2006.21.2.355.

[11] J. L. Zha, Average shadowing property and pseudo-orbit tracing property, Appl. Math. Ser. B. 3 (2004) 311-314. https://doi.org/10.2116/analsci.20.717.

[12] Y. W. Dong, X. T. Tian, X. P. Yuan, Ergodic properties of systems with asymptotic average shadowing property, J. Math. Anal. Appl. 432 (2015) 53-73. https://doi.org/10.1016/j.jmaa.2015.06.046. 